\title{
A DNA Nano-Tweezer for Intracellular Detection of HER2 mRNA by Confocal Microscopy
}

\author{
Haiying Que ${ }^{1, a}$, Fei Mo ${ }^{1, b}$, Tong Wang ${ }^{1, c}$, Xiaoyu Yan ${ }^{1, d}$, Hongmin Ma ${ }^{1, e}$ and Yurong Yan,f,* \\ ${ }^{1}$ Key Laboratory of Clinical Laboratory Diagnostics (Ministry of Education), College of Laboratory \\ Medicine, Chongqing Medical University, Chongqing 400016, China \\ fyanyurong163@163.com \\ *corresponding author
}

Keywords: HER2 mRNA, DNA nano-tweezer, breast carcinoma

\begin{abstract}
DNA nanostructure plays an important role in intracellular detection strategies for its good biocompatibility and nontoxicity. Herein, we design a DNA nano-tweezer with aptamer and fluorophores to detect HER2 mRNA in living breast carcinoma cells. The designed nano-tweezer is self-assembled with ten DNA strands. It has one head and two arms with a long hangover each. A HER2 aptamer in its head is for targeting tumor cell and two overhangs with fluorophores can hybridize with HER2 mRNA. Following, the as-prepared nano-tweezer can effectively enter cancer cells by specific cross-talking between aptamers and overexpressed cell-surface receptors. Finally, the HER2 mRNA fluorescence detection is achieved by a confocal microscope, and the fluorescence intensity indicates mRNA expression quantity. Thus, this strategy provides a useful platform for efficient detection of HER2 mRNA in living cells.
\end{abstract}

\section{Introduction}

In breast carcinomas, the amplification of the HER2 gene induces protein overexpression in the cell membrane, which plays an important role in aggressive human breast cancer. [1-4] Since cancer cells express diverse surface receptors (such as anti-nucleolin aptamer), [5] the DNA nano-tweezer can internalize in cancer cells by cell surface receptors mediated endocytosis. By using WatsonCrick base-pairing, a variety of DNA nanostructures have been self-assembled, which show the advantages of flexible design, orientation, excellent biocompatibility, and demonstrate significant potential for clinic application in biosensing, early stage cancer diagnosis and targeted therapies.[69] In this work, the specific HER2 aptamer is self-assembled in nano-tweezer to ensure highly efficient and specific cell targeting based on surface receptors expressed on tumor cells. By smart designing of the DNA strands, the two hangovers of nano-tweezer can bind to mRNA, resulting conversion of nano-tweezer from closed state to open state. Thus, the fluorophore is separated from quencher, achieving fluorescence imaging of the HER2 mRNA expression. This constructed nanotweezer is proved to be a simple and highly efficient platform for detection of HER2 mRNA in living cells.

\section{Experimental}

\subsection{Reagents}

All DNA oligonucleotides used in this work were synthesized and purified using highperformance liquid chromatography by Sangon Inc. (Shanghai, China). Dulbecco's Modified Eagle Medium (DMEM) and Fetal Bovine Serum (FBS) were obtained from Gibco Inc. (Shanghai, China). Diethylpyrocarbonate (DEPC) was purchased from Solarbio Inc. (Beijing, China). The human breast cancer cell lines including SK-BR-3 and MDA-MB-231 cells used in this work were obtained from the American Type Culture Collection (ATCC). (Rockville, MD, USA). The DNA strands were dissolved in Tank buffer (pH 7.5, $20 \mathrm{mM}$ Tris, $125 \mathrm{mM} \mathrm{NaCl}, 20 \mathrm{mM} \mathrm{KCl}$ ) and stored 
at $-20{ }^{\circ} \mathrm{C}$ prior to use. All other reagents were of analytical grade, and Millipore-Q water $(\geq 18 \mathrm{M} \Omega)$ was used in all experiments.

\subsection{Instruments}

Fluorescence signal was recorded by an Agilent Cary Eclipse fluorescence spectrophotometer (Agilent Technologies, USA). Immunofluorescence and confocal imaging were performed on a Nikon A1R Confocal Microscope (Nikon, Japan). The concentration of all RNA and DNA oligonucleotides were measured by a Thermo NanoDrop ${ }^{\mathrm{TM}} 1000$ Spectrophotometer (Thermo Fisher Scientific, USA).

\subsection{Fluorescence detection}

DNA nano-tweezer was prepared with $0.8 \mu \mathrm{M}$ of DNA strands from 1 to 10 in TAE/ $\mathrm{Mg}^{2+}$ buffer (pH 8.0, $40 \mathrm{mM}$ Tris, $20 \mathrm{mM}$ acetic acid, $2 \mathrm{mM}$ EDTA and $7.5 \mathrm{mM}$ magnesium acetate, $20 \mathrm{mM}$ potassium acetate). All samples were annealed in a thermostatic water bath for 5 min at $95{ }^{\circ} \mathrm{C}$ and naturally cooled to room temperature. Cells were cultured in DMEM supplemented with 10\% FBS, penicillin $(100 \mathrm{U} / \mathrm{mL})$ and streptomycin $(100 \mu \mathrm{g} / \mathrm{mL})$ at $37{ }^{\circ} \mathrm{C}$ in a humidified incubator $\left(5 \% \mathrm{CO}_{2}\right.$ and $95 \%$ air). Cells ( $3 \times 10^{5}$ per well) were plated into 6-well plates for $12 \mathrm{~h}$. Subsequently, the cells were added DNA nano-tweezer and stained immunofluorescence (DAPI for Cell nuclei) in different wells as indicted. Fluorescence images were collected by a Nikon A1R Confocal Microscope.

\section{Results and discussion}

The DNA nano-tweezer was annealed with $0.8 \mu \mathrm{M}$ of DNA strands from 1 to 10 . Here we used SK-BR-3 (breast cancer cells) as an in vitro model system.

As shown in Figure 1, DNA nano-tweezer was tagged with fluorescein isothiocyanate (FITC) and Cyanine3 (Cy3) at each arm. There was strong fluorescence intensity in the presence of DNA nano-tweezer than that of without DNA nano-tweezer, suggesting that DNA nano-tweezer entered cancer cells successfully.
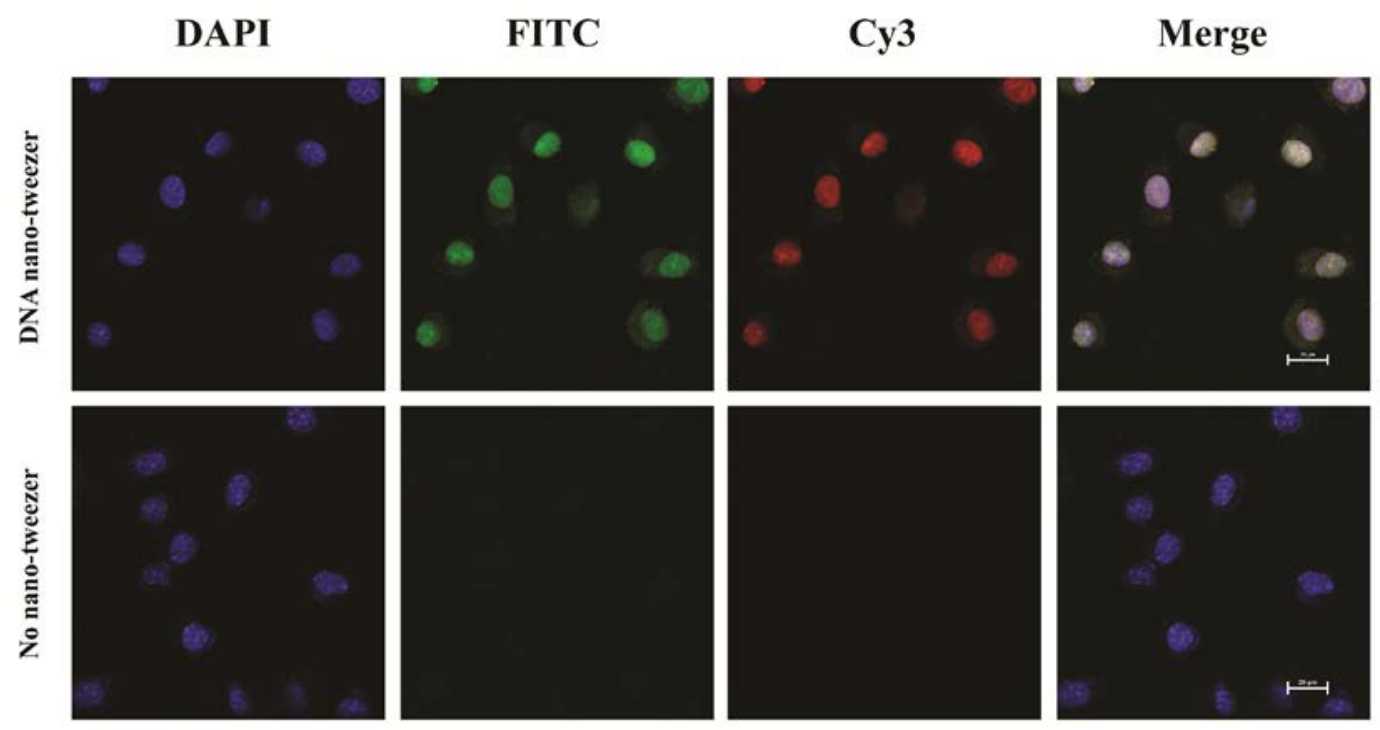

Figure 1. Confocal fluorescence microscopic images of SK-BR-3 cells incubated with and without

DNA nano-tweezer. The cell nucleus was stained with DAPI. The merge image shows colocalization of both fluorophores in cytoplasm of SK-BR-3 cells. Scale bars: $20 \mu \mathrm{m}$

As shown in Figure 2, DNA nano-tweezer was only tagged with FITC, the effect of the reaction time was also evaluated; the maximum fluorescence intensity value was achieved at $2 \mathrm{~h}$. 


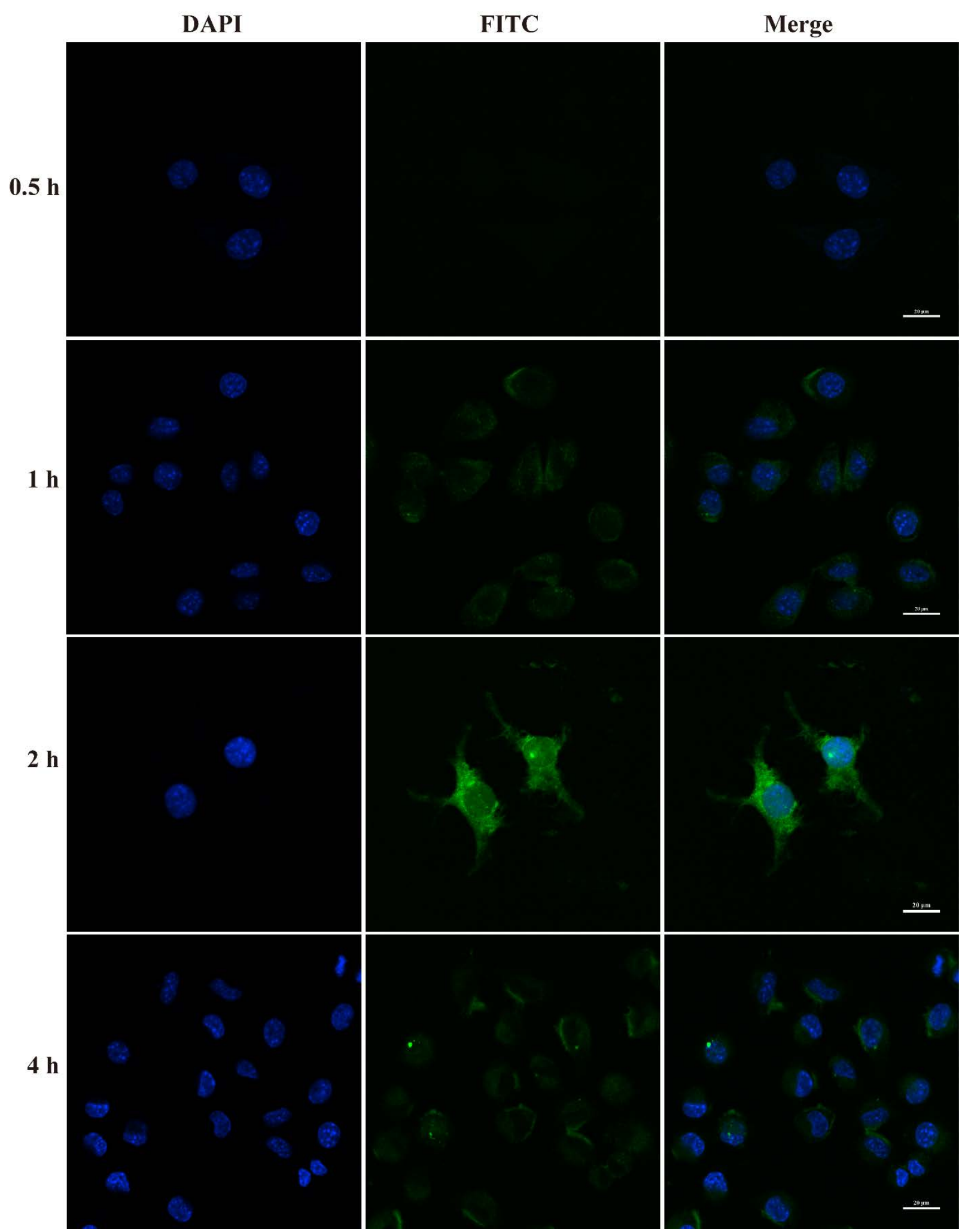

Figure 2. Confocal microscopy images of SK-BR-3 cells after incubation with $0.5 \mu \mathrm{M}$ DNA nano-tweezer for $0.5 \mathrm{~h}, 1 \mathrm{~h}, 2 \mathrm{~h}$ and $4 \mathrm{~h}$. Scale bars: $20 \mu \mathrm{m}$

As shown in Figure 3, the recognition and uptake of DNA nano-tweezer were further verified with two different cells, SK-BR-3 cells as positive group, and MDA-MB-231 cells as negative group. The bright fluorescence was only observed within SK-BR-3 cells. Only little scattered fluorescence was observed from MDA-MB-231 cells, demonstrating the specific recognition and high-efficiency delivery of DNA nano-tweezer to target cells. 
DAPI
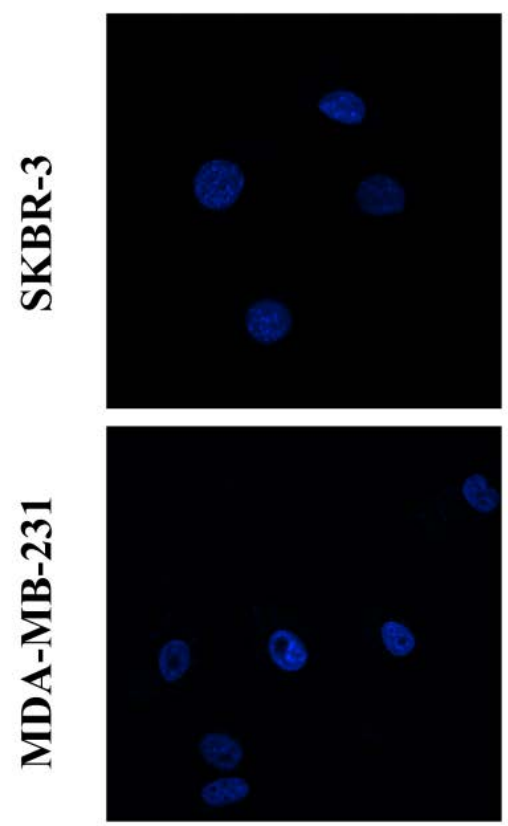

FITC
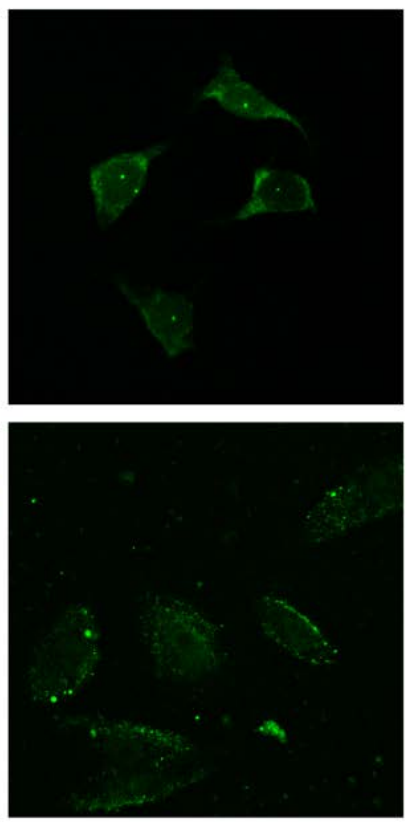

Merge
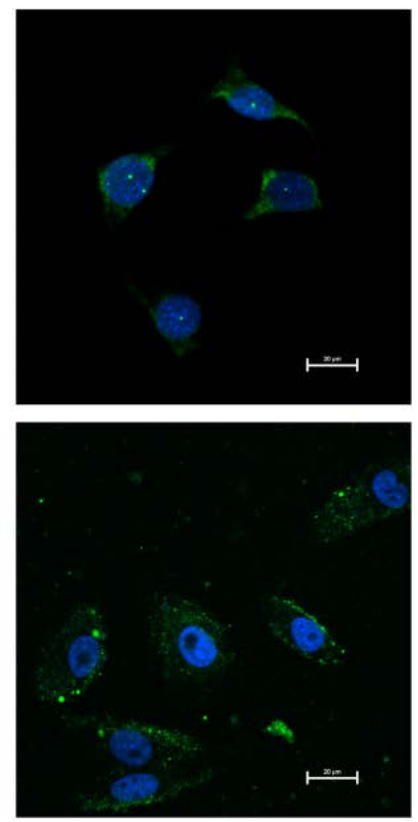

Figure 3. Specificity of DNA nano-tweezer induced translocation. Confocal microscopy images of SK-BR-3 cells and MDA-MB-231 cells after incubation with $0.5 \mu \mathrm{M}$ DNA nano-tweezer for $2 \mathrm{~h}$.

Scale bars: $20 \mu \mathrm{m}$

\section{Conclusion}

In summary, we design a DNA nano-tweezer with aptamer and fluorophores to detect HER2 mRNA in living breast carcinoma cells. The as-prepared rigid structure DNA nano-tweezer with defined geometry (both length and diameter) can effectively enter cancer cells by specific crosstalking between aptamers and overexpressed cell-surface receptors. The results demonstrate that the nano-tweezer shows promise for intracellular delivery vehicle. Thus, this strategy provides a useful platform for efficient detection of HER2 mRNA in living cells.

\section{Acknowledgements}

This work was funded by the National Natural Science Foundation of China (81371904).

\section{References}

[1] Wu, C., Han, D., Chen, T., Peng, L., Zhu, G., You, M., Qiu, L., Sefah, K., Zhang, X. and Tan, W. (2013) Building a Multifunctional Aptamer-Based DNA Nanoassembly for Targeted Cancer Therapy. Journal of the American Chemical Society, 135, 18644-18650.

[2] Pei, H., Zuo, X., Zhu, D., Huang, Q. and Fan, C. (2014) Functional DNA Nanostructures for Theranostic Applications. Accounts of Chemical Research, 47, 550-559.

[3] Lee, J.B., Hong, J., Bonner, D.K., Poon, Z. and Hammond, P. T. (2012) Self-Assembled RNA Interference Microsponges for Efficient siRNA Delivery. Nature Materials, 11, 316.

[4] Jo, H., Her, J. and Ban, C. (2015) Dual Aptamer-Functionalized Silica Nanoparticles for the Highly Sensitive Detection of Breast Cancer. Biosensors and Bioelectronics, 71, 129-136. 
[5] Hu, Y., Duan, J., Cao, B., Zhang, L., Lu, X., Wang, F., Yao, F., Zhu, Z., Yuan, W., Wang, C. and Yang, X.D. (2015) Selection of a Novel DNA Thioaptamer Against HER2 Structure. Clinical and Translational Oncology, 17, 647-656.

[6] Douglas, S.M., Bachelet, I. and Church, G.M. (2012) A Logic-Gated Nanorobot for Targeted Transport of Molecular Payloads. Science, 335, 831-834.

[7] Chen, Y.J., Groves, B., Muscat, R.A. and Seelig, G. (2015) DNA Nanotechnology from the Test Tube to the Cell. Nature Nanotechnology, 10, 748-760.

[8] Zhang, Z., Jiao, Y., Zhu, M. and Zhang, S. (2017) Nuclear-Shell Biopolymers Initiated by Telomere Elongation for Individual Cancer Cell Imaging and Drug Delivery. Analytical Chemistry, 89, 4320-4327.

[9] Bujold, K.E., Hsu, J.C. and Sleiman, H.F. (2016) Optimized DNA "Nanosuitcases" for Encapsulation and Conditional Release of siRNA. Journal of the American Chemical Society, 138, 14030-14038. 